\title{
Persistent Chlamydia Pneumoniae serology is related to decline in lung function in women but not in men. Effect of persistent Chlamydia pneumoniae infection on lung function
}

\author{
Thorarinn Gislason ${ }^{1,2}$, Vilmundur Guðnason ${ }^{1,3}$, Bryndis Benediktsdottir ${ }^{1}$, Isleifur Olafsson ${ }^{4}$, Thor Aspelund ${ }^{1,3}$,
} Bjarni Thjodleifsson ${ }^{1,2}$, Christer Janson ${ }^{5^{*}}$

\begin{abstract}
Background: Chlamydia pneumoniae (C pn) infection causes an acute inflammation in the respiratory system that may become persistent, but little is known about the long-term respiratory effects of $C$ pn infections. Aim: To estimate the long term respiratory effects of $C \mathrm{pn}$ with change in forced expiratory volume in one second (FEV $\left.{ }_{1}\right)$ and forced vital capacity (FVC) as a main outcome variable.

Methods: The study comprised of 1109 subjects (500 men and 609 women, mean age $28 \pm 6$ years) that participated in the Reykjavik Heart Study of the Young. Spirometry and blood samples for measurements of lgG antibodies for $C$ pn were done at inclusion and at the end of the follow-up period (mean follow-up time $27 \pm 4$ years).

Results: Having lgG against $C$ pn at both examinations was significantly associated to a larger decrease in FEV ( $6 \mathrm{~mL} /$ year) and FVC (7 mL/year) in women but not in men. In women the association between $\mathrm{C}$ pn and larger $\mathrm{FEV}_{1}$ decline was only found in women that smoked at baseline where having C pn IgG was associated with 10 $\mathrm{mL} /$ year decline compared to smokers without $\mathrm{C} \mathrm{pn} \mathrm{lgG.} \mathrm{These} \mathrm{results} \mathrm{were} \mathrm{still} \mathrm{significant} \mathrm{after} \mathrm{adjustment} \mathrm{for}$ age, smoking and change in body weight.

Conclusion: Our results indicate that persistent $C$ pn serology is related to increased decline in lung function in women but not in men. This effect was, however, primarily found in smoking women. This study is a further indication that the pathophysiological process leading to lung impairment may differ between men and women.
\end{abstract}

\section{Background}

Chlamydia pneumoniae (C pn) is an intracellular gramnegative pathogen that is detected in 5 to $10 \%$ of community-acquired pneumonia and other lower respiratory tract infections [1] Most adults are infected at least once during their lifetime, as indicated by seroprevalence of 70 to $80 \%$ [2]. C pn respiratory diseases may manifest as an acute disease or persistent and recurring infection that causes intense chronic inflammation. Growing evidence indicates that inflammation results from cellular responses by non immune cells, including mucosal

\footnotetext{
* Correspondence: christer.janson@medsci.uu.se

${ }^{5}$ Respiratory Medicine and Allergology, Uppsala University, Uppsala, Sweden Full list of author information is available at the end of the article
}

epithelial and vascular endothelial cells [3]. Studies have suggested that $\mathrm{C}$ pn may be related to the pathogenesis of wheeze in children [4], asthma in adults [5] and to chronic obstructive pulmonary disease (COPD) [6]. Systemic aspects of COPD include oxidative stress and altered circulating levels of inflammatory mediators and acute-phase proteins. C-reactive protein (CRP) reflects the total systemic burden of inflammation in several disorders and has been shown to up regulate the production of proinflammatory cytokines [7]. Systemic inflammation is increasingly being recognised as a risk factor for a number of different complications including atherosclerosis, cachexia, anorexia, and osteoporosis, but all of these complications are commonly observed in 
patients with COPD [8]. Associations between C pn serology and atherosclerosis [9] and ischemic heart diseases have been reported, as well as an additive or synergistic effect of other persistent infections on atherosclerosis [10]. These cardiovascular associations with persistent infections may be highly relevant in COPD since ischemic heart disease and stroke are the leading causes of mortality among patients with COPD [8].

Several studies have reported gender differences in the association between risk factors and pulmonary diseases. In one study of young children a positive serology for $\mathrm{C}$ pn was related to wheeze in girls but not in boys [4]. Other studies have shown gender differences in the association between various other risk factors and changes in lung function such as smoking [11] and CRP [12]. Most previous studies on the association of $C$ pn and respiratory diseases have been performed on patient samples from different clinical settings, but large population-based longitudinal studies are lacking. No studies are available on the association between $\mathrm{C}$ pn serology and long term changes in lung function.

The primary aim of the present research was to study the association between $C$ pn serology and changes in lung function in a longitudinal population study with particular focus on gender differences.

\section{Methods}

\section{The Reykjavik Study of the Young}

The Reykjavík Study of the Young was conducted in 3 stages between 1973 and 2003 and recruited 2147 participants aged 25-62 years. Stages 1,2 and 3 took part in the years: 1 (1973-1974); 2 (1983-1985); 3 (2001-2003). The present research is based on a subset of the Reykjavik Study of the Young which consisted of a random sample of individuals born in 1940 through 1954 who were living in the greater Reykjavik in 1973. The aim of the study was to compare younger generations to the older ones who were being investigated in the Reykjavik Study $[12,13]$

The present study focuses on the pulmonary part of the database, the methodology of which has been described separately [13]. Only subjects who had participated both at baseline (either stage 1 or 2) and followup (stage 3) were included in the present analysis. In addition two serum samples and acceptable pulmonary testing were needed. Altogether 1109 came for the follow-up study in 2001-2003, of whom 962 had come for the first stage in 1973-74 and 147 in 1983-85. The lung function tests included measurements of forced expiratory volume in one second $\left(\mathrm{FEV}_{1}\right)$ and forced vital capacity (FVC).

Smoking status was recorded at each survey. Participants were divided into never smokers at baseline, current or ex-smokers, and the number of pack years was calculated. Body mass index (BMI) was calculated as weight in kilograms divided by the square of height in meters.

\section{Serologic methods}

IgG antibodies against Chlamydia pneumoniae (C pn) were measured using reagents from the Immuno Biological Laboratories, Hamburg, Germany. Serum samples that gave indefinite results in antibody measurements were classified as seronegative.

\section{Statistics}

Statistical analyses were performed using STATA 9 software (Stata Corp., Texas, USA). The Chi ${ }^{2}$ test and unpaired $t$ test were used to compare characteristics between men and women. Multiple linear regression was used to analyse the association between lung function and C pn IgG status. A p-value $<0.05$ was considered as statistically significant. In these regression analyses change in $\mathrm{FEV}_{1}$ and FVC from baseline to follow up ( $\mathrm{mL} /$ year) was used as the dependent variable. The analyses were stratified by sex and the following independent variables were included: age (mean age between the surveys), age $^{2}$, height, BMI, change in BMI and pack years between the surveys.

\section{Ethics}

Approval was obtained from The National Bioethics Committee and The Data Protection Authority in Iceland

\section{Results}

The characteristics of the study population are presented in Table 1. Women were more likely to be smokers at baseline and had a higher smoking exposure during the follow-up. Women were leaner at baseline but had a larger increase in BMI during the follow-up period. Men were more likely to have IgG antibodies against $\mathrm{C}$ pn at both surveys (Figure 1).

\section{$C$ pn serology and change in lung function}

Women with $\mathrm{C}$ positive $\mathrm{C}$ pn serology at both examinations had a larger decline in $\mathrm{FEV}_{1}$ and FVC than women with negative $C$ pn serology, while no corresponding association was found in men. Women that developed positive $\mathrm{C}$ pn serology during the follow-up had a significantly larger decline in FVC, whereas the corresponding association to $\mathrm{FEV}_{1}$ was of border-line significance $(\mathrm{p}=0.06)$ (Table 2). Decline in lung function was associated with an increase in BMI and smoking in both men and women, while a higher baseline BMI was associated with a greater decline in FVC but not in $\mathrm{FEV}_{1}$. 
Table 1 Characteristics of the participants ( $\%$ and mean \pm SD)

\begin{tabular}{|c|c|c|c|c|}
\hline & $\begin{array}{l}\text { Men } \\
(n=500)\end{array}$ & $\begin{array}{l}\text { Women } \\
(n=609)\end{array}$ & p-value & $\begin{array}{l}\text { All } \\
(n=1109)\end{array}$ \\
\hline Age at baseline & $27.9 \pm 5.7$ & $27.4 \pm 5.4$ & 0.15 & $27.6 \pm 5.6$ \\
\hline Smoking history (\%) & & & $<0.0001$ & \\
\hline never & 41.8 & 41.5 & & 41.7 \\
\hline ex & 23.6 & 14.1 & & 18.4 \\
\hline current & 34.6 & 44.3 & & 40.0 \\
\hline Pack years between surveys in current smokers & $14.7 \pm 13.1$ & $17.0 \pm 12.5$ & 0.04 & $15.8 \pm 12.9$ \\
\hline BMl kg/m2 & $23.2 \pm 2.8$ & $22.0 \pm 3.1$ & $<0.0001$ & $22.5 \pm 3.0$ \\
\hline Change in BMl over 10 yrs. & $1.5 \pm 1.2$ & $1.9 \pm 1.3$ & $<0.0001$ & $1.7 \pm 1.3$ \\
\hline
\end{tabular}

\section{Interactions}

The sex difference in association between change in lung function and $\mathrm{C}$ pn serology was statistically significant for FEV1 ( pinteraction $=0.04$ ) and almost significant for FVC ( $\left.p_{\text {interaction }}=0.08\right)$. A significant interaction was found between women that were non-smokers and smokers at baseline $\left(p_{\text {interaction }}=0.02\right)$. Women that were smokers had a significantly larger decline in lung function if they had a positive $\mathrm{C}$ pn serology at the second or at both examinations (Figure 2). No such corresponding interaction was found for men (Figure 3). No interactions were found concerning the association between $\mathrm{C}$ pn and FVC. No interactions were found for BMI or birth cohort concerning the association between $\mathrm{C}$ pn and change in lung function.

\section{Discussion}

The main result of the study was that $C$ pn infections were associated with increased lung function decline. This finding was, however, only seen in women. This study thereby provides further evidence of sex differences in the mechanisms related to decline in lung function. Our study also indicates that $\mathrm{C}$ pn may enhance the lung damaging effect of smoking in women.

The main strength of our study is that it is a population-based longitudinal study with a follow-up of 27 years. A potential weakness is that IgG antibodies measured by ELISA were used as the only indicator of persistent infection with $\mathrm{C}$ pn. Previous studies have shown that high levels of IgG antibodies to $C$ pn measured by ELISA do not persist for half a decade after

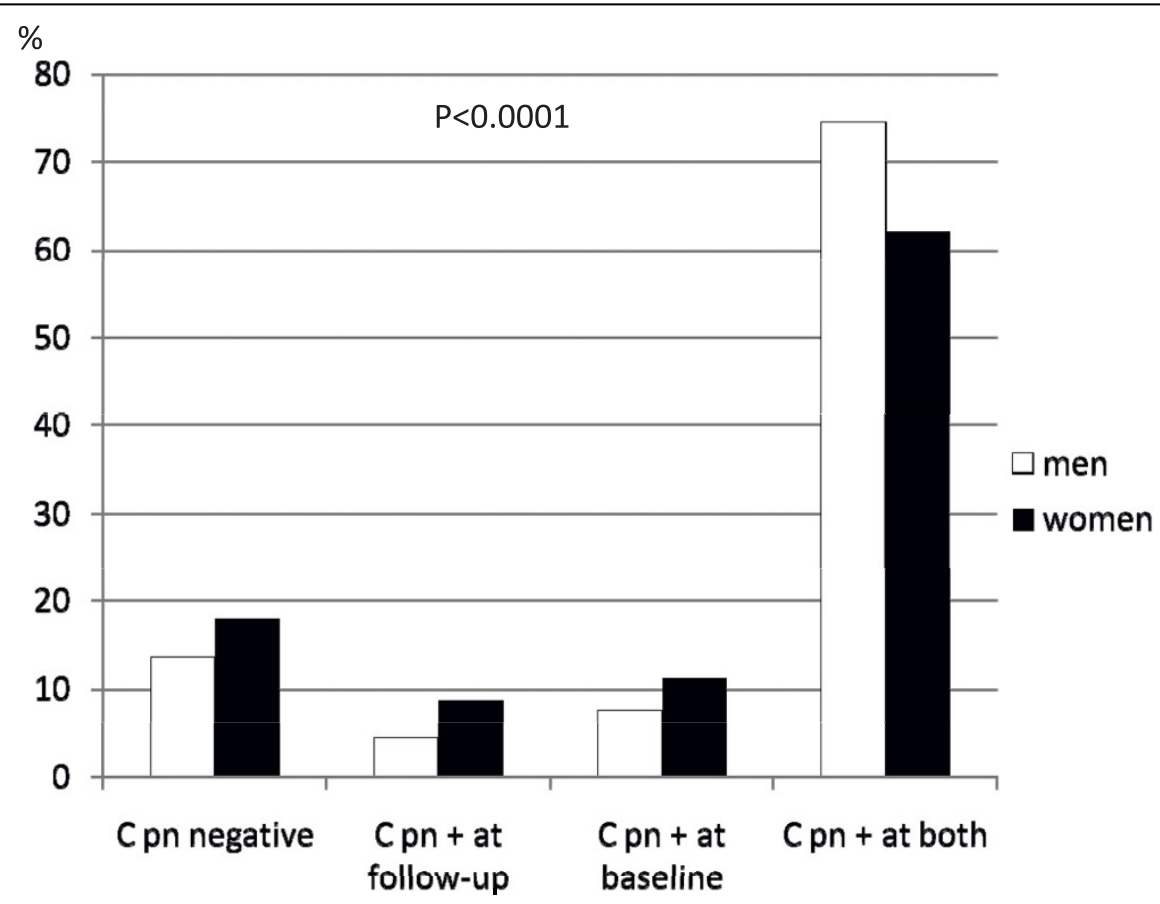

Figure 1 Proportion men and women divided after Chlamydia pneumoniae (C pn) serology at baseline and follow-up. 
Table 2 Association between C pn serology and change in FEV1 and FVC (mL/year) in men and women

\begin{tabular}{|c|c|c|c|c|}
\hline & \multicolumn{2}{|c|}{ Men } & \multicolumn{2}{|c|}{ Women } \\
\hline & FEV1 & FVC & FEV1 & FVC \\
\hline C pn only at follow up & $-1.8(-11,7.5)$ & $-7.0(-16,2.5)$ & $-5.6(-11,0.3)$ & $-7.4(-12,-2.6)$ \\
\hline C pn only at baseline & $2.6(-5.0,10)$ & $-1.4(-9.1,6.3)$ & $-0.5(-5.9,5.0)$ & $-1.6(-6.0,2.8)$ \\
\hline $\mathrm{C}$ pn at both examinations & $2.1(-2.9,7.0)$ & $-0.3(-5.3,4.8)$ & $-4.5(-8.2,-0.7)$ & $-4.4(-7.5,-1.3)$ \\
\hline $\begin{array}{l}\text { BMI at baseline } \\
\text { (per } 5 \text { units) }\end{array}$ & $0.6(-2.6,3.9)$ & $-5.1(-8.5,-1.8)$ & $-1.1(-3.5,1.3)$ & $-4.3(-6.2,-2.3)$ \\
\hline$\triangle \mathrm{BMI}$ (per 10 years) & $-2.1(-3.5,-0.4)$ & $-5.3(-6.9,-3.7)$ & $-2.2(-3.2,-1.1)$ & $-2.8(-3.6,-1.9)$ \\
\hline Pack years between surveys (per 10 units) & $-2.1(-3.5,-0.7)$ & $-3.0(-4.4,-1.5)$ & $-1.6(-2.7,-0.5)$ & $-2.0(-2.9,-1.0)$ \\
\hline
\end{tabular}

The estimates are adjusted for the variables in the table and age, height and BMI.

seroconversion without reinfection or reactivation [14]. In young military recruits IgG antibody levels measured by microimmunofluorescence test decrease rapidly after the infection [15]. The microimmunofluorescence test is however not suitable for seroepidemiological studies. Two studies have demonstrated that the ELISA test has comparable sensitivity and specificity to the microimmunofluorescence technique $[14,16]$. Another possible weakness is that our study population was not assessed by post-bronchodilator lung function testing and we can not know how much of the decline in FEV1 was due to reversible (asthma) or irreversible airflow obstruction (COPD). The widespread use of postbronchodilator spirometry is mostly confined to the twenty-first century. The long follow-up time of our study population is, however, unique.

The finding that $\mathrm{C}$ pn infections are more strongly related to lung impairment in women than in men fit surprisingly well with data from children where an association between wheeze and C pn IgG was stronger in girls than in boys [4]. There are several reports on gender differences in the association between wheezing and asthma irrespective of $\mathrm{C}$ pn or other infections. The predominant trend reported is a greater incidence of wheezing and asthma in boys with a reversal between ages 10-20 when the incidence becomes greater in females [17-22]. Girls are reported to be more vulnerable than boys to the impact of smoking and overweight on respiratory symptoms and lung function [23]. In contrast to the findings in children, Chinn et al found that weight gain had a larger effect in men than in women [11]. A stronger association between systemic inflammation and lung function decline in men than in women has also been reported in several studies [12,24,25]. Several mechanisms have been suggested to explain these gender differences. The sexes may develop their airway disease based on different sex related genetics[26] or on different immunological time scales [27]. Female sex

\section{Women}
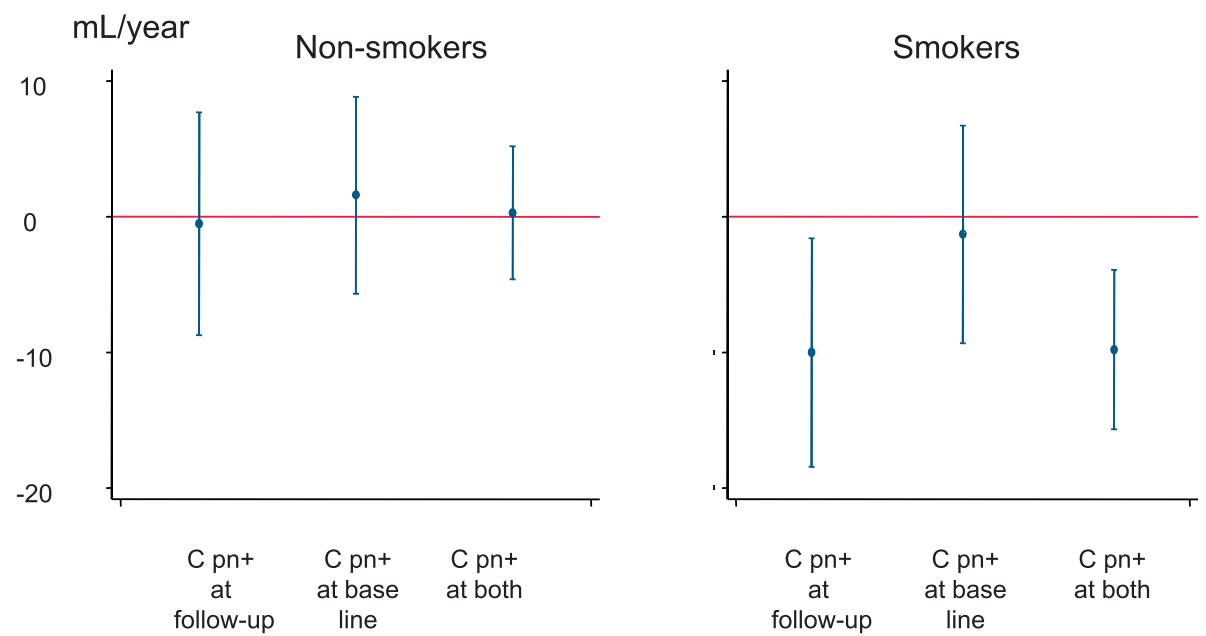

Figure 2 Association between Chlamydia pneumoniae ( $\mathrm{C}$ pn) serology and change in $\mathrm{FEV}_{1}$ ( $\mathrm{mL} /$ year) in women that are non-smokers or current smokers. The estimates are adjusted for age, height, smoking (pack years), BMI and change in BMl. Participants that were C pn negative at both surveys are the reference group in each smoking status group. 


\section{Men}
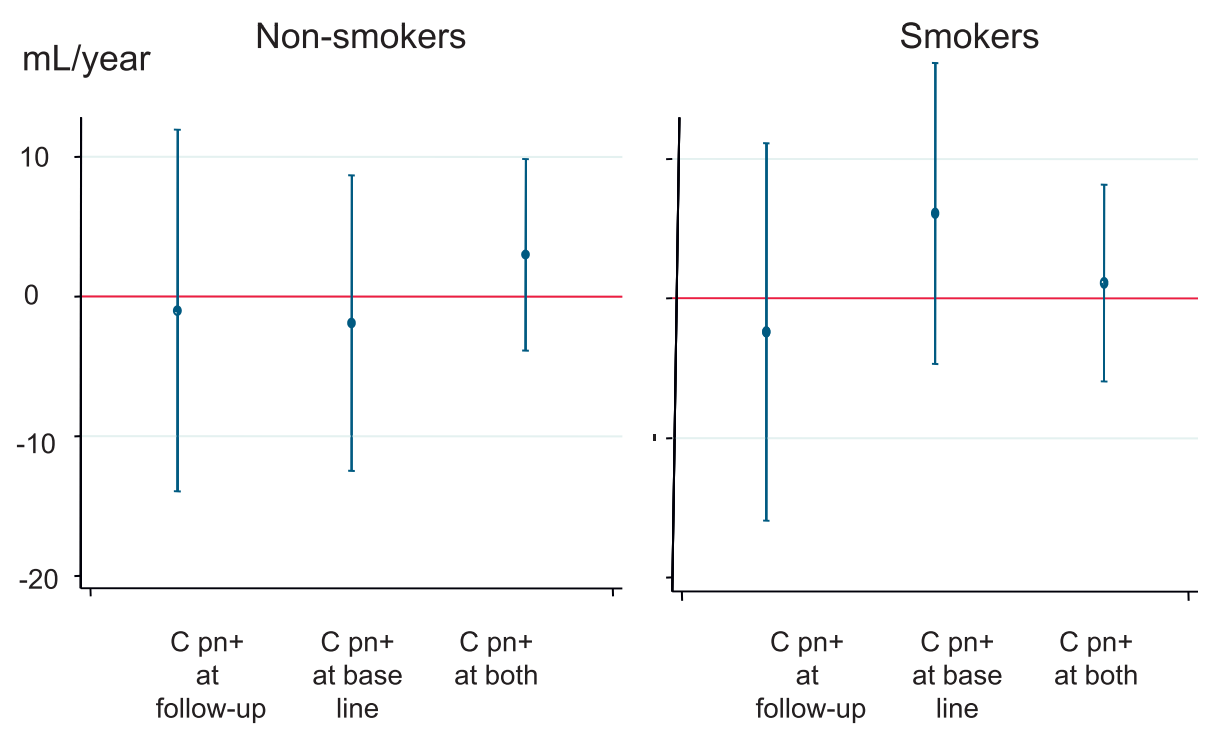

Figure 3 Association between Chlamydia pneumoniae ( $\mathrm{C}$ pn) serology and change in $\mathrm{FEV}_{1}$ ( $\mathrm{mL} / \mathrm{year}$ ) in men that are non-smokers or current smokers. The estimates are adjusted for age, height, smoking (pack years), BMI and change in BMI. Participants that were C pn negative at both surveys are the reference group in each smoking status group.

hormones may also be part of the explanation for the gender difference as there is increasing evidence that sex hormones play a role in lung function development and decline. For an instance, it has been recently bee reported that girls with an early menarche have lower lung function as adults [28].

There are reports on a positive association of $\mathrm{C} \mathrm{pn}$ and IgA and IgG serology and COPD $[29,30]$ but negative associations have also been reported [31]. A causal association between $\mathrm{C}$ pn and COPD has, therefore, not been proven and possible mechanisms are not clear[32]. In a recent experimental study an intranasal inoculation with $C$. pneumoniae on day 0 was from day 7 associated with both sustained bronchial hyper-responsiveness and airway inflammation in mice [33]. An association between bronchial hyperresponsiveness and IgA antibodies against $C$ pn has also been found in Swedish population study [34]. Acute in vitro experiments of human lung tissue with $C$ pn suggest that $C$ pn plays different roles during acute and chronic stages of pulmonary infection [35].

If persistent $\mathrm{C}$ pn infections play a significant role in the pathogenesis of asthma, COPD and lung function decline, this opens the therapeutic possibility of useful antibiotic treatment The data supporting antibiotic therapy are limited, however, as shown in a Cochrane review of macrolide usage in treatment of chronic asthma [36] and further long term studies are needed to confirm the possible role of persistent infections in the decline in lung function.

\section{Conclusion}

In conclusion, our results indicate that persistent $C$ pn serology is related to increased decline in lung function in women but not in men. This effect was, however, primarily found in smoking women. This study is a further indication that the pathophysiological process leading to lung impairment may differ between men and women.

\section{Abbreviations}

BMI: body mass index; COPD: chronic obstructive pulmonary disease; C pn: Chlamydia pneumoniae; FEV1: forced expiratory volume in one second; FVC: forced vital capacity (FVC); IgA: immunoglobulin A; IgG: immunoglobulin G

\section{Acknowledgements}

This study was supported financially by Icelandic Research Council grant no. 050405011 and The Landspitali-University Hospital Research Fund.

\section{Author details}

${ }^{1}$ University of Iceland, Faculty of Medicine, Reykjavik, Iceland. ${ }^{2}$ Department of Medicine Landspitali University Hospital, Reykjavik, Iceland. ${ }^{3}$ Icelandic Heart Association, Kopavogur, Iceland. ${ }^{4}$ Department of Clinical Biochemistry, Landspitali University Hospital, Reykjavik, Iceland. ${ }^{5}$ Respiratory Medicine and Allergology, Uppsala University, Uppsala, Sweden.

\section{Authors' contributions}

TG, BT and BB drafted the manuscript. VG was responsible for the clinical investigation, and data collection. 10 carried out the serological measurements. TA was responsible for data management. CJ drafted the manuscript and carried out the statistical analyses. All authors read and approved the final manuscript.

\section{Competing interests}

The authors declare that they have no competing interests.

Received: 8 April 2010 Accepted: 25 August 2010 Published: 25 August 2010 


\section{References}

1. Hammerschlag MR: Chlamydia pneumoniae and the lung. Eur Respir J 2000, 16:1001-1007.

2. Leinonen M: Pathogenetic mechanisms and epidemiology of Chlamydia pneumoniae. Eur Heart J 1993, 14(Suppl K):57-61.

3. Stephens RS: The cellular paradigm of chlamydial pathogenesis. Trends Microbiol 2003, 11:44-51.

4. Normann E, Gnarpe J, Wettergren B, Janson C, Wickman M, Nordvall L: Association between Chlamydia pneumoniae antibodies and wheezing in young children and the influence of sex. Thorax 2006, 61:1054-1058.

5. Hahn DL, Dodge RW, Golubjatnikov R: Association of Chlamydia pneumoniae (strain TWAR) infection with wheezing, asthmatic bronchitis, and adult-onset asthma. JAMA 1991, 266:225-230.

6. Grayston JT, Campbell LA, Kuo CC, Mordhorst CH, Saikku P, Thom DH, et al: A new respiratory tract pathogen: Chlamydia pneumoniae strain TWAR. J Infect Dis 1990, 161:618-625.

7. Karadag F, Kirdar S, Karul AB, Ceylan E: The value of C-reactive protein as a marker of systemic inflammation in stable chronic obstructive pulmonary disease. Eur J Intern Med 2008, 19:104-108.

8. Hansell AL, Walk JA, Soriano JB: What do chronic obstructive pulmonary disease patients die from? A multiple cause coding analysis. Eur Respir $J$ 2003, 22:809-814.

9. Arcari CM, Gaydos CA, Nieto FJ, Krauss M, Nelson KE: Association between Chlamydia pneumoniae and acute myocardial infarction in young men in the United States military: the importance of timing of exposure measurement. Clin Infect Dis 2005, 40:1123-1130

10. Jha HC, Vardhan H, Gupta R, Varma R, Prasad J, Mittal A: Higher incidence of persistent chronic infection of Chlamydia pneumoniae among coronary artery disease patients in India is a cause of concern. BMC Infect Dis 2007, 7:48

11. Chinn S, Jarvis D, Melotti R, Luczynska C, Ackermann-Liebrich U, Anto JM, et al: Smoking cessation, lung function, and weight gain: a follow-up study. Lancet 2005, 365:1629-1635.

12. Olafsdottir IS, Gislason T, Thjodleifsson B, Olafsson I, Gislason D, Jogi R, et al: Gender differences in the association between C-reactive protein, lung function impairment, and COPD. Int J Chron Obstruct Pulmon Dis 2007, 2:635-642.

13. Chinn S, Gislason T, Aspelund T, Gudnason V: Optimum expression of adult lung function based on all-cause mortality: results from the Reykjavik study. Respir Med 2007, 101:601-609.

14. Paldanius MBA, Alho M, Leinonen M, Saikku P: Prevalence and persistence of Chlamydia pneumoniae antibodies in healthy laboratory personnel in Finland. Clin Diagn Lab Immunol 2005, 12:654-659.

15. Paldanius $M$, Juvonen $R$, Leinonen $M$, Bloigu A, Silvennoinen-Kassinen S, Saikku P: Asthmatic persons are prone to the persistence of Chlamydia pneumoniae antibodies. Diagn Microbiol Infect Dis 2007, 59:117-122.

16. Romano Carratelli CNI, Cozzolino D, Bentivoglio CPR, Rizzo A: Relationship between Chlamydia pneumoniae infection, inflammatory markers, and coronary heart diseases. International Immunopharmacology 2006, 6:848-853.

17. de Marco R, Locatelli F, Sunyer J, Burney P: Differences in incidence of reported asthma related to age in men and women. $A$ retrospective analysis of the data of the European Respiratory Health Survey. Am J Respir Crit Care Med 2000, 162:68-74.

18. Nicolai T, Pereszlenyiova-Bliznakova L, Illi S, Reinhardt D, von Mutius E: Longitudinal follow-up of the changing gender ratio in asthma from childhood to adulthood: role of delayed manifestation in girls. Pediatr Allergy Immunol 2003, 14:280-283.

19. Toren $\mathrm{K}$, Hermansson BA: Incidence rate of adult-onset asthma in relation to age, sex, atopy and smoking: a Swedish population-based study of 15813 adults. Int J Tuberc Lung Dis 1999, 3:192-197.

20. Brogger J, Bakke P, Eide GE, Johansen B, Andersen A, Gulsvik A: Long-term changes in adult asthma prevalence. Eur Respir J 2003, 21:468-472.

21. Thomsen SF, Ulrik CS, Larsen K, Backer V: Change in prevalence of asthma in Danish children and adolescents. Ann Allergy Asthma Immunol 2004, 92:506-511.

22. Kurukulaaratchy RJ, Matthews S, Arshad SH: Relationship between childhood atopy and wheeze: what mediates wheezing in atopic phenotypes? Ann Allergy Asthma Immunol 2006, 97:84-91.
23. Tollefsen E, Bjermer L, Langhammer A, Johnsen R, Holmen TL: Adolescent respiratory symptoms-girls are at risk: the Young-HUNT study. Norway. Respir Med 2006, 100:471-476.

24. Thorleifsson SJ, Margretardottir OB, Gudmundsson G, Olafsson I, Benediktsdottir B, Janson C, Buist AS, Gislason T: Chronic airflow obstruction and markers of systemic inflammation: Results from the BOLD study in Iceland. Respir Med 2009, 103:1548-1553.

25. Ólafsdóttir IS, Janson C, Lind L, Hulthe J, Gunnbjörnsdóttir M, Sundström J: Serum MMP-9, TIMP-1 and their ratio is associated with impaired lung function in the elderly - a population-based study. Respirol 2010, 15:530-535.

26. Melen E, Kere J, Pershagen G, Svartengren M, Wickman M: Influence of male sex and parental allergic disease on childhood wheezing: role of interactions. Clin Exp Allergy 2004, 34:839-844.

27. Hansbro PM, Beagley KW, Horvat JC, Gibson PG: Role of atypical bacteria infection of the lung in predisposition/protection of asthma. Pharmaco Ther 2004, 101:193-210.

28. Macsali F, Gómez Real F, Plana E, Sunyer J, Anto JM, Dratva J, Janson C, Jarvis D, Omenaas ER, Zemp E, Wjst M, Leynaert B, Svanes C: Age of menarche, lung function and adult asthma. Am J Respir Crit Care Med 2010.

29. Branden E, Koyi H, Gnarpe J, Gnarpe H, Tornling G: Chronic Chlamydia pneumoniae infection is a risk factor for the development of COPD. Respir Med 2005, 99:20-26.

30. Falck G, Gnarpe J, Hansson LO, Svardsudd K, Gnarpe H: Comparison of individuals with and without specific IgA antibodies to Chlamydia pneumoniae: respiratory morbidity and the metabolic syndrome. Chest 2002, 122:1587-93

31. Mendall MA, Carrington D, Strachan D, Patel P, Molineaux N, Levi J, et al: Chlamydia pneumoniae: risk factors for seropositivity and association with coronary heart disease. J Infect 1995, 30:121-128.

32. Blasi F, Tarsia P, Aliberti S: Chlamydophila pneumoniae. Clin Microbiol Infect 2009, 15:29-35

33. Blasi F, Aliberti S, Allegra L, Piatti G, Tarsia P, Ossewaarde JM, et al: Chlamydophila pneumoniae induces a sustained airway hyperresponsiveness and inflammation in mice. Respir Res 2007, 8:83.

34. Björnsson E, Hjelm E, Janson C, Fridell E, Boman G: Serology of Chlamydia relation to asthma and bronchial hyperresponsiveness. Scand J Infect Dis 1996, 28:63-69.

35. Droemann D, Rupp J, Goldmann T, Uhlig U, Branscheid D, Vollmer E, et al: Disparate innate immune responses to persistent and acute Chlamydia pneumoniae infection in chronic obstructive pulmonary disease. Am J Respir Crit Care Med 2007, 175:791-797.

36. Richeldi L, Ferrara G, Fabbri LM, Lasserson TJ, Gibson PG: Macrolides for chronic asthma. Cochrane Database Syst Rev 2005, 4: CD002997.

\section{Pre-publication history}

The pre-publication history for this paper can be accessed here: http://www.biomedcentral.com/1471-2466/10/44/prepub

doi:10.1186/1471-2466-10-44

Cite this article as: Gislason et al:: Persistent Chlamydia Pneumoniae serology is related to decline in lung function in women but not in men. Effect of persistent Chlamydia pneumoniae infection on lung function. BMC Pulmonary Medicine 2010 10:44.

\section{Submit your next manuscript to BioMed Central and take full advantage of:}

- Convenient online submission

- Thorough peer review

- No space constraints or color figure charges

- Immediate publication on acceptance

- Inclusion in PubMed, CAS, Scopus and Google Scholar

- Research which is freely available for redistribution

Submit your manuscript at www.biomedcentral.com/submit
C Biomed Central 